$54^{\text {ème }}$ Congrès de la SFMBCB, 03002 (2011)

DOI: $10.1051 / \mathrm{sfmbcb} / 20115403002$

(C) Owned by the authors, published by EDP Sciences, 2011

\title{
Lymphome plasmoblastique associé au virus de l'immunodéficience humaine : présentation d'un cas
}

\author{
Boisramé-Gastrin $\mathbf{S}^{\mathbf{1}}$, Hansart V, Quintin Roué I, Prédine-Hug $\mathbf{F}^{\mathbf{1}}$, Tempescul A \\ ${ }^{1}$ UFR d'Odontologie, Service d'Odontologie, CHU, Brest, France \\ ${ }^{2}$ Service d'Hématologie, CHU, Brest, France \\ ${ }^{3}$ Laboratoire d'Anatomo-Pathologie, CHU, Brest, France \\ sylvie.boisrame-gastrin@chu-brest.fr
}

Le lymphome plasmoblastique est une variante des lymphomes diffus à grandes cellules $\mathrm{B}$, observé surtout dans la cavité orale chez le patient immunodéprimé. Il a été décrit pour la première fois par Delacluse et al. en 1997. L'immunophénotypie se caractérise par une faible expression des antigènes CD19, CD20 et CD45 par les cellules B et par une forte expression des antigènes CD38 et CD138 par les cellules plasmocytaires (Folk et al. 2006). Le traitement du lymphome plasmoblastique n'est pas standardisé. Plusieurs approches thérapeutiques ont été proposées et les résultats restent modestes.

Le cas rapporté concerne un patient âgé de 43 ans, séropositif pour le VIH depuis dix ans, et présentant un lymphome plasmoblastique siégeant sur la branche horizontale gauche de la mandibule. L'examen anatomo-pathologique montre de larges lymphocytes avec une apparence immmunoblastique, l'immunophénotypage montre des cellules négatives aux antigènes CD3, CD10, CD20, CD30 mais positives aux antigènes CD38 et CD138. Le CT-scan corps entier n'a retrouvé aucune autre localisation. L'examen de la moelle osseuse était normal. En première intention le patient a reçu la thérapie HAART (Highly Active Anti-Retroviral Therapy) associée à des inhibiteurs d'intégrase. Le traitement du lymphome a comporté plusieurs lignes de chimiothérapie : anthracycline, agents alkylants, cytarabine et médicaments immunomodulateurs avec une efficacité discutable. L'association DHAOx (Dexamethasone, Cytarabine, Oxaliplatine)lénalidomide a entrainé une régression de $75 \%$ de la lésion initiale mais une rechute est survenue après la troisième cure. Pendant la chimiothérapie, le taux de CD4 était inférieur à $50 . \mathrm{mm}^{-3}$. Le traitement a été complété par une radiothérapie localisée qui a permis d'obtenir une rémission complète. Huit semaines après la fin de la radiothérapie, il y a eu une récidive sous la forme de multiples lésions cutanées nodulaires, associées à une cruralgie gauche inflammatoire. Le CT-scan corps entier a mis en évidence de multiples lésions hépatiques, spléniques, pancréatiques, pulmonaires, une volumineuse masse pré-coccygienne, et des adénopathies médiastinales et coeliomésentériques. Le patient est décédé un an après la découverte de son lymphome, dans un contexte de thrombopénie profonde, persistante associée à une hémorragie digestive haute et un tableau d'occlusion intestinale.

Le lymphome plasmoblastique est une forme rare de lymphome, très agressive, avec une survie globale de 12 à 22 mois. Il n'y a pas de traitement standardisé. Plusieurs protocoles de chimiothérapie ont été proposés mais leur efficacité est controversée (Colomo et al. 2004). Dans le cas rapporté, aucun des traitements réalisés n'a pas été efficace, conformément aux données de la littérature : la réponse au traitement est associée au score IPI mais aussi à la réponse virale à la thérapie HAART (Ribera et al. 2008). Cependant, le succès initial de la chimiothérapie DHAOx associée à la lénalidomide et à de la radiothérapie, parait intéressant.

This is an Open Access article distributed under the terms of the Creative Commons Attribution-Noncommercial License 3.0, which permits unrestricted use, distribution, and reproduction in any noncommercial medium, provided the original work is properly cited. 